\title{
Second Primary Neoplasms in Patients With Uveal Melanoma: A SEER Database Analysis
}

\author{
INÊS LAÍNS, CARLA BARTOSCH, VERA MONDIM, BRIAN HEALY, IVANA K. KIM, DEEBA HUSAIN, AND \\ JOAN W. MILLER
}

- PURPOSE: To determine the risk of second primary neoplasms (SPNs) in subjects previously diagnosed with uveal melanoma (UM), including an analysis on whether radiotherapy is a risk factor to develop these SPNs.

- DESIGN: Retrospective cohort study.

- METHODS: Using the Surveillance, Epidemiology, and End Results (SEER) 9 database, we identified patients diagnosed with UM as their first malignancy between 1973 and $2011(\mathrm{n}=3976)$. We obtained standardized incidence ratios (SIR) and excess absolute risks of SPNs on patients with UM compared to a reference population. Multivariate Cox regression models were used to evaluate the effect of radiotherapy in SPN risk.

- RESULTS: Sixteen percent $(n=641)$ of the patients developed SPNs during a median follow-up of 83 months (range, 1-463 months). This represented an 11\% excess risk compared to the reference population, mainly owing to a significantly increased risk of skin melanomas $(\mathrm{SIR}=2.93,95 \% \mathrm{CI}: 2.23-3.78)$ and kidney tumors $(\mathrm{SIR}=1.91,95 \% \mathrm{CI}: 1.27-2.76)$, primarily in those diagnosed between 30 and 59 years of age. The occurrence of second UM was also increased (SIR $=16.90$, 95\% CI: 9.00-28.90), which likely includes recurrences misclassified as a second cancer. Radiotherapy was performed in $39 \%(n=1538)$ of the patients. Multivariate analysis revealed that this treatment was not an independent risk factor for SPNs (hazard ratio $=1.06,95 \%$ CI: $0.88-1.26, P=.54)$.

- CONCLUSIONS: Patients with UM presented an $11 \%$ higher risk of SPNs compared to the reference population. Radiotherapy does not seem to be a risk factor. SPNs should be considered in the surveillance of UM. (Am J Ophthalmol 2016;165:54-64. (C) 2016 Elsevier Inc. All rights reserved.)

\footnotetext{
AJO.com Supplemental Material available at AJO.com. Accepted for publication Feb 19, 2016.

From the Department of Ophthalmology, Massachusetts Eye and Ear Infirmary, Harvard Medical School, Boston, Massachusetts (I.L., I.K.K., D.H., J.W.M.); Faculty of Medicine, University of Coimbra, Coimbra, Portugal (I.L.); Department of Pathology, Portuguese Oncology Institute, Porto, Portugal (C.B.); Department of Anesthesiology, Centro Hospitalar de Lisboa Central, EPE, Lisbon, Portugal (V.M.); and Biostatistics Center, Massachusetts General Hospital, Boston, Massachusetts (B.H.).

Inquiries to Deeba Husain, Department of Ophthalmology, Massachusetts Eye and Ear Infirmary, Harvard Medical School, 243 Charles St, Boston, MA 02114; e-mail: Deeba_Husain@meei.harvard.edu
}

$\mathrm{P}$ UBLISHED DATA ON SURVIVAL OF UVEAL MELAnoma demonstrate that more than half of the patients are long-term survivors. At 5 and 10 years, the survival rates range between $68 \%-82 \%$ and $57 \%-$ $62 \%$, respectively. ${ }^{1-3}$ It is well established that cancer survivors are especially prone to developing independent second primary neoplasms (SPNs) and that their characteristics vary according to the site of the first primary tumor. ${ }^{4,5}$ This increased risk is recognized to have multifactorial causes. Lifestyle and environmental exposures, such as smoking ${ }^{6}$ and alcohol consumption, ${ }^{7}$ have been implicated. Additionally, host susceptibility factors, including genetic risk factors, ${ }^{5,8}$ also seem to play a role. Besides the well-established relevance of genetic susceptibility for cancer in general, SPNs can be part of familial cancer predisposition syndromes. Interestingly, one has been recently described for uveal melanoma. Associated with germline BAP1 mutations, it includes a constellation of uveal and cutaneous melanoma, among other tumors. ${ }^{9-12}$

The relevance of the treatment modalities applied to the first tumor has also been highlighted, in particular for radiation therapy. ${ }^{13,14}$ This is especially important, as radiotherapy is currently the gold-standard treatment for most uveal melanomas. ${ }^{15,16}$ The suggested underlying factors for SPN risk after this treatment include the doses and modalities applied, as well as the volume of tissue irradiated. ${ }^{4,5}$ This risk is most pronounced in the organs within the irradiated fields ${ }^{13,14}$ but has also been described in sites not directly exposed to radiation. ${ }^{17,18} \mathrm{~A}$ potential explanation includes "bystander response-like phenomena," ${ }^{19,20}$ through the release of blood cells and mediators from the irradiated fields that can induce genomic instability and cell transformation. ${ }^{18,21}$

Despite growing knowledge about treatment-induced effects on the occurrence of SPNs in patients with other malignancies, ${ }^{8}$ data are insufficient for uveal melanoma. A few population-based studies ${ }^{22-25}$ were published before radiotherapy became the treatment of choice for these tumors, and therefore did not address this question. More recently, the Collaborative Ocular Melanoma Study (COMS) Group ${ }^{26}$ reported the occurrence of SPN in patients treated for choroidal melanoma, characterizing the time to occurrence of SPN and the most frequently affected sites. However, no data from the general population were included.

We present a population-based study that aimed to evaluate whether patients with uveal melanoma demonstrate 
an increased incidence of SPNs compared to the general population, including an analysis on whether radiation therapy is associated with a higher risk of these SPNs.

\section{METHODS}

- STUDY POPUlATION: This retrospective cohort study followed a group of patients with a diagnosis of uveal melanoma as their first malignancy, who were identified in the Surveillance, Epidemiology, and End Results (SEER) 9 database from 1973 to $2011 .^{27}$ The SEER program collects data on all persons diagnosed with cancer residing in several geographically defined regions-the SEER 9 database includes 9 registries, covering approximately $10 \%$ of the United States (US) population and being comparable to the US general population, with a case ascertainment rate of $98 \%$. Despite including fewer registries than the newer SEER databases, SEER 9 is the only one that provides data from 1973 onwards. ${ }^{27}$ This study met the definition of exempt research. As a result, Institutional Review Board approval was not required. The SEER data is considered nonhuman subject research. The data is anonymized. No patient contact is involved in its collection. The collection of SEER data is also exempt from Health Insurance Portability and Accountability Act regulations.

Patient selection was based on the primary site and histologic subtype of the first malignancy, according to the International Classification of Diseases for Oncology, third edition (ICD-O-3), as follows: topography codes C69.2 (retina), C69.3 (ciliary body), and C69.4 (choroid) for uvea; morphology codes 8720.3 to 8774.3 for melanoma. As previously described, ${ }^{3}$ most "retinal" melanomas probably represent misclassification of uveal melanoma; thus they were also considered. ICD-O-3 includes the iris in ciliary body topography. Therefore, these cases were not analyzed separately. SPNs were considered as those diagnosed at least 2 months after the uveal melanoma, as defined by the SEER program. ${ }^{27}$ We excluded cases where the diagnosis of uveal melanoma was only made by death certificate or autopsy, ${ }^{28}$ because they cannot contribute with persontime for SPN assessment. For both primary and SPNs, we selected only cases with malignant behavior. Regarding treatment for the first malignancy, the SEER database includes information on the type of radiotherapy provided (ie, beam radiation, radioactive implants/radioisotopes, combination of both, radiation not specified or unknown). No details are available for the type of beam irradiation applied and for the doses of any of these treatment modalities. Additionally, there are also no data in the SEER database on the type of surgery performed (ie, enucleation or other surgical modalities) for eye malignancies.

- STATISTICAL ANALYSIS: We used the SEER*Stat program - MP-SIR session (Surveillance Research Program,
National Cancer Institute SEER*Stat software; seer. cancer.gov/seerstat, version 8.1.5) to perform the multiple primary analyses. Person-years at risk were determined starting in the third month after uveal melanoma diagnosis until date of death, last follow-up, SPN diagnosis, or end of 2011, whichever came first. Observed number of cancers corresponded to the actual reported SPN cases. The expected number of SPNs corresponded to those that would develop if the study cohort patients experienced the same rate of occurrence as a reference SEER population, with comparable sex, 5-year age group, race (white/unknown, black, other), site, and calendar year. For this analysis, we used the reference population "Incidence - SEER 9 Regs Research Data, Nov 2013 Sub (1973-2011) <Katrina/ Rita Population Adjustment $>$ - Linked To County Attributes - Total U.S., 1969-2012 Counties." Briefly, this reference population includes all people living in the SEER 9 geographic areas between 1973 and 2011, based on the US Census Bureau's Population Estimates Program External Web Site Policy, in collaboration with the National Center for Health Statistics, and with support from the NCI through an interagency agreement. ${ }^{29}$ Referent rates (for expected cases) represent SEER 9 incidence rates, which are then stratified by age, sex, race, calendar year, and cancer site, according to the characteristics of our cohort of uveal melanoma patients.

Standardized incidence ratios (SIR) were obtained by observed-to-expected ratio and the $95 \%$ confidence interval $(95 \% \mathrm{CI})$ was calculated based on the Poisson distribution. The excess absolute risk of SPN was calculated by subtracting the expected number from the number of observed cases and dividing by the person-years at risk, and corresponded to the additional cancers (beyond the expected amount). It was expressed per 10000 person-years.

Univariate and multivariate Cox proportional hazards regression models were used to evaluate the effect of radiotherapy on SPN risk. Owing to the small number of nonwhite subjects in our cohort, for these analyses race was coded as a dichotomous variable (white/unknown vs black/other). A secondary analysis investigated the effect of the type of radiotherapy (beam radiation, radioactive implants/radioisotopes) on SPN risk. For this analysis, subjects given a combination of radiotherapy or with unknown type of radiotherapy were excluded. For multivariate analysis, the proportional hazards assumption was tested using the $\log$ minus $\log$ curve $\{\ln [\ldots \ln (S)]\}$ and the goodness-of-fit test, and no violation was observed for any of the variables. Stata version 12.1 (StataCorp LP, College Station, Texas, USA) was used for all these Cox analyses. The previous analyses censored all subjects who died at the time of death, but an alternative approach could consider death from uveal melanoma or death from other cause as a competing risk. Therefore, all of the previous multivariate models were refit accounting for competing risks using the stcompet and stcrreg routines in Stata. 
TABLE 1. Demographic and Clinical Characteristics of the Population-based Cohort $(n=3976)$ of Patients With Uveal Melanoma

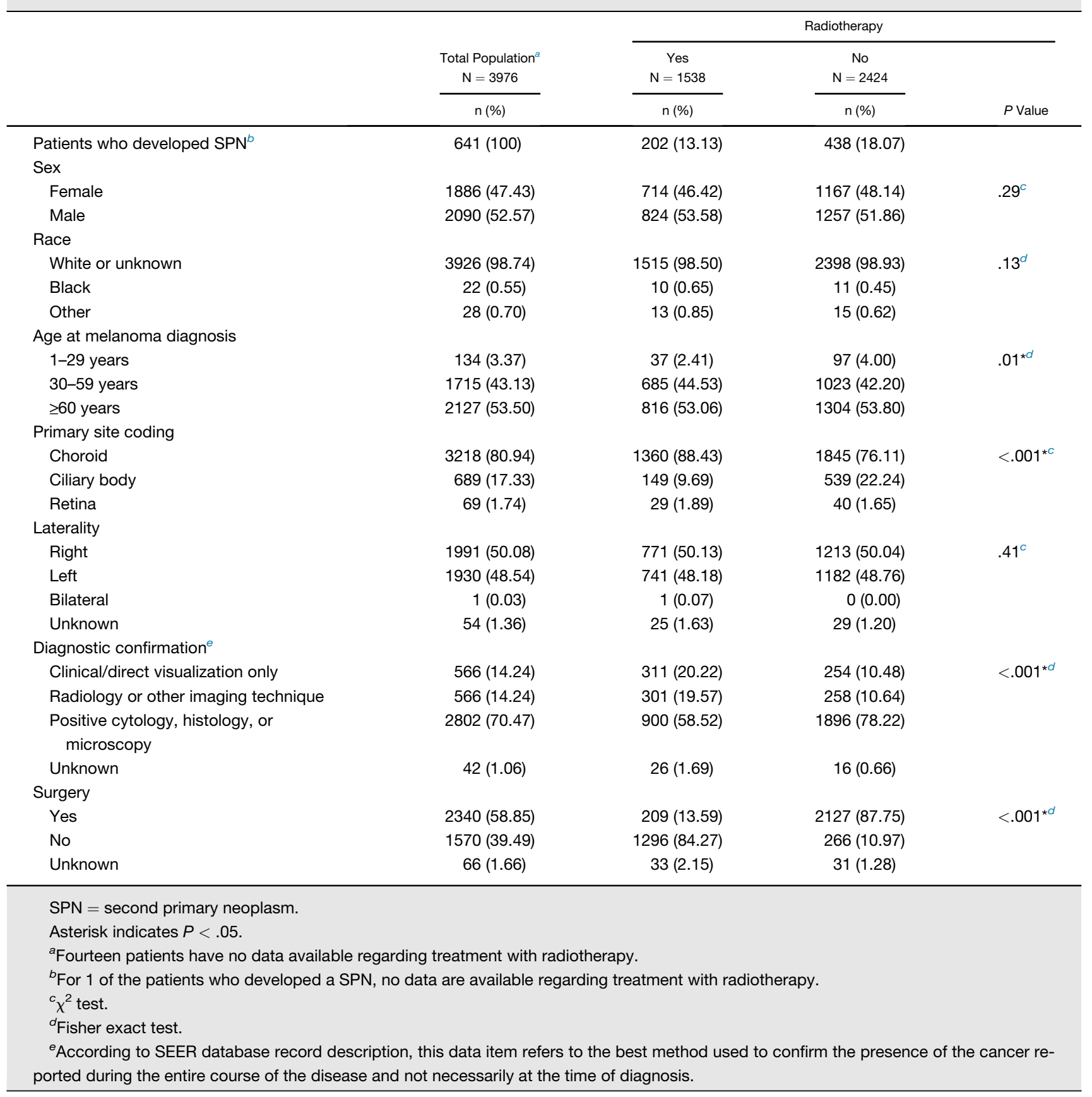

\section{RESULTS}

- STUDY POPULATION: The study cohort consisted of 3976 patients diagnosed with uveal melanoma as their first malignancy (Table 1). Most cases were coded as malignant melanoma without other histologic specification $(\mathrm{n}=$ $2475,62 \%)$. The remaining cases were classified as spindle cell melanoma ( $\mathrm{n}=768,19 \%)$, epithelioid cell melanoma ( $\mathrm{n}=192,5 \%)$, mixed epithelioid and spindle cell melanoma $(\mathrm{n}=504,13 \%)$, amelanotic melanoma $(\mathrm{n}=32$,
$0.8 \%)$, and others $(\mathrm{n}=5,0.1 \%)$. The median follow-up time was 83 months, with $62 \%(n=2427)$ of the subjects followed for at least 5 years (range, $1-463$ months). Nearly $30 \%(\mathrm{n}=1168)$ of the patients died of uveal melanoma and the 5- and 10-year disease-specific survival rates were $80 \%$ and $69 \%$, respectively. The global survival rate during the available follow-up was $44 \%(n=1740)$.

- SECONDARY CANCERS AFTER UVEAL MELANOMA: SPN occurred in 641 of the patients with uveal melanoma 


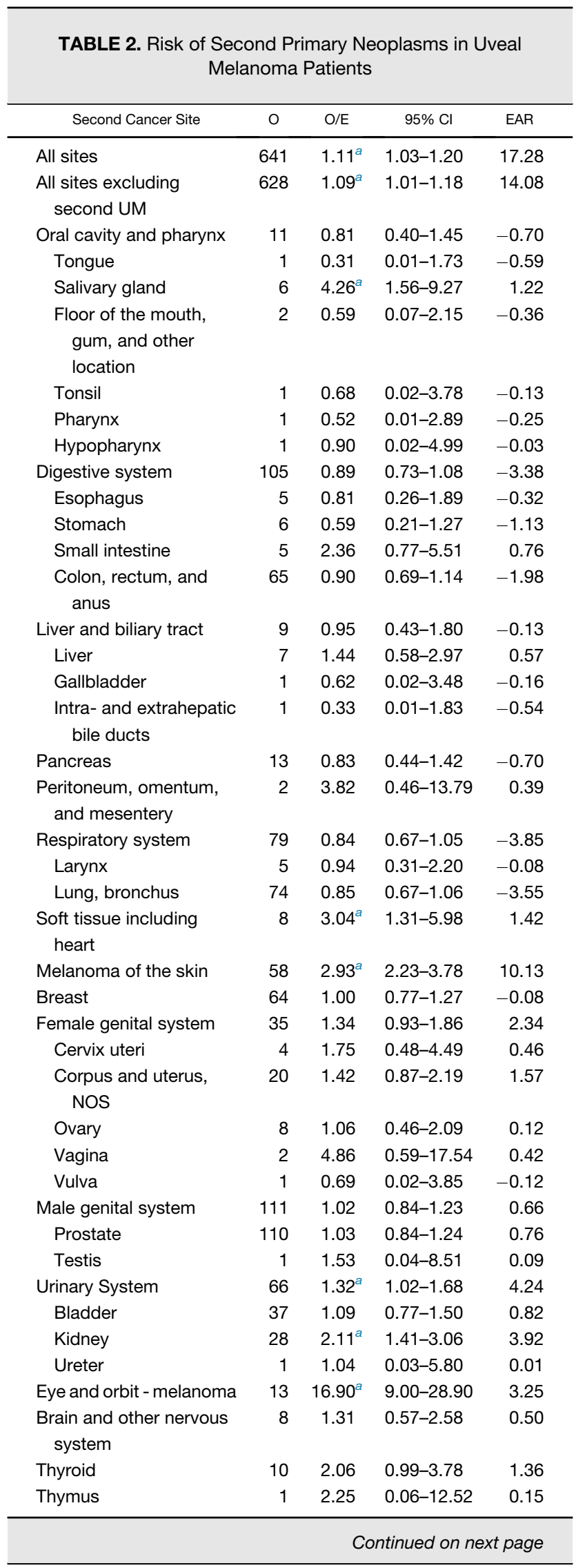

\begin{tabular}{|c|c|c|c|c|}
\hline \multicolumn{5}{|c|}{$\begin{array}{l}\text { TABLE 2. Risk of Second Primary Neoplasms in Uveal } \\
\text { Melanoma Patients (Continued) }\end{array}$} \\
\hline Second Cancer Site & 0 & O/E & $95 \% \mathrm{Cl}$ & EAR \\
\hline $\begin{array}{l}\text { Lymphatic and } \\
\text { hematopoietic } \\
\text { diseases }\end{array}$ & 48 & 1.00 & $0.74-1.33$ & -0.01 \\
\hline Lymphoma & 18 & 0.74 & $0.44-1.17$ & -1.66 \\
\hline Myeloma & 10 & 1.35 & $0.65-2.48$ & 0.68 \\
\hline Leukemia & 20 & 1.22 & $0.75-1.89$ & 0.96 \\
\hline Mesothelioma & 2 & 1.18 & $0.14-4.27$ & 0.08 \\
\hline Miscellaneous & 20 & 1.41 & $0.86-2.18$ & 1.58 \\
\hline \multicolumn{5}{|c|}{$\begin{array}{l}\mathrm{Cl}=\text { confidence interval; } \mathrm{EAR}=\text { excess absolute risk; } \mathrm{O}= \\
\text { observed number of cases; } \mathrm{O} / \mathrm{E} \text { ratio }=\text { observed over expected } \\
\text { number of cases; } \mathrm{UM}=\text { uveal melanoma. } \\
\text { When observed values were equal to } 0 \text {, expected values were } \\
\text { reported. No correction for multiple comparisons was performed } \\
\text { for any of the above analyses. } \\
{ }^{a} 95 \% \mathrm{Cl} \text { not including the null value }(=1) \text {, so } P<.05 \text {. }\end{array}$} \\
\hline
\end{tabular}

(16\%), within a median time of 75 months (range, 2425 months). Most ( $\mathrm{n}=583,15 \%)$ had 1 additional cancer and a few had $2(\mathrm{n}=57,1 \%)$ or $3(\mathrm{n}=1,0.03 \%)$ new primaries. SIRs demonstrated that patients with uveal melanoma have a significant $11 \%$ excess risk of SPN when compared to the reference population, with an excess absolute risk of $17.28 / 10000$ person-years. This was mainly owing to a significantly higher risk of tumors of the salivary glands, kidney, and soft tissues, as well as skin melanomas (Table 2). The median age at the time of diagnosis of these SPNs was 77, 67, 81, and 69 years, respectively.

A statistically significant increased risk for a second uveal melanoma was also noticed in our study cohort, with 13 cases reported (Table 3) within a median latency time of 7 years (range, 2 months to 27 years). Two of these cases occurred in the fellow eye, 2 months and 7 years later than the first malignancy. Ipsilateral second melanomas were coded as new primaries in 9 patients and in 2 patients data regarding laterality were not available. Most cases of second uveal melanomas ( $\mathrm{n}=9,69 \%)$ occurred after an initial treatment with radiotherapy. As discussed below, we considered that at least some of these second tumors likely represent misclassified recurrences. Therefore, we recalculated the primary outcomes with these second malignancies excluded. Our results confirmed that patients with uveal melanoma demonstrated an increased risk of SPN for all sites of 1.09 (95\% CI: 1.01-1.18), excluding cases coded as a second uveal melanoma (Table 2).

When evaluating the risk during the follow-up period (Table 4), we observed that the increased risk for salivary gland tumors was only significant within the first 5 years after the diagnosis of uveal melanoma. Kidney tumors occurred more frequently in 2 periods: in the first 5 years 
TABLE 3. Characteristics of Patients With a Second Uveal Melanoma Coded as a New Primary

\begin{tabular}{|c|c|c|c|c|c|c|c|c|c|c|}
\hline \multirow[b]{2}{*}{ Patient } & \multirow[b]{2}{*}{ Age } & \multicolumn{5}{|c|}{ First uveal melanoma } & \multicolumn{4}{|c|}{ Second uveal melanoma } \\
\hline & & Year of Diagnosis & Site Code & Histology & Eye & Therapy & Year of Diagnosis & Site Code & Histology & Eye \\
\hline 1 & 57 & 1973 & Choroid & Malign. melanoma, NOS & OD & $\begin{array}{l}\text { Radiation, } \\
\text { NOS }\end{array}$ & 1980 & Choroid & Malign. melanoma, NOS & OS \\
\hline 2 & 47 & 1994 & Choroid & Malign. melanoma, NOS & $O D$ & BT & 2003 & Choroid & SCM, NOS & OD \\
\hline 3 & 50 & 2001 & $\mathrm{CB}$ & Malign. melanoma, NOS & OD & $\mathrm{BR}$ & 2009 & Choroid & Mixed epithelioid and SCM & OD \\
\hline 4 & 69 & 1991 & Choroid & Malign. melanoma, NOS & NA & $\mathrm{BR}$ & 1995 & Choroid & Malign. melanoma, NOS & OS \\
\hline 5 & 54 & 1997 & Choroid & Malign. melanoma, NOS & OD & BT & 2002 & Choroid & Mixed epithelioid and SCM & OD \\
\hline 6 & 57 & 2002 & Choroid & Malign. melanoma, NOS & OD & BT & 2002 & Choroid & Malign. melanoma, NOS & os \\
\hline 7 & 60 & 2005 & Choroid & SCM, type B & OD & Surgery & 2006 & Eye, NOS & Malign. melanoma, NOS & OD \\
\hline 8 & 30 & 1974 & $\mathrm{CB}$ & Malign. melanoma, NOS & OD & Surgery & 1999 & $\mathrm{CB}$ & Epithelioid cell melanoma & OD \\
\hline 9 & 32 & 1978 & $\mathrm{CB}$ & Malign. melanoma, NOS & os & Surgery & 1999 & $\mathrm{CB}$ & Malign. melanoma, NOS & NA \\
\hline 10 & 53 & 2008 & Choroid & Malign. melanoma, NOS & OS & BT & 2011 & Choroid & SCM, type B & os \\
\hline 11 & 71 & 1998 & Choroid & Malign. melanoma, NOS & OD & BT & 2004 & Choroid & Mixed epithelioid and SCM & OD \\
\hline 12 & 71 & 2002 & Choroid & Malign. melanoma, NOS & OD & $\mathrm{BR}+\mathrm{BT}$ & 2005 & Orbit, NOS & Mixed epithelioid and SCM & OD \\
\hline 13 & 61 & 1981 & Choroid & Mixed epithelioid and SCM & OS & Surgery & 2008 & Orbit, NOS & Malign. melanoma, NOS & OS \\
\hline
\end{tabular}

$\mathrm{BR}=$ beam radiation; $\mathrm{BT}=$ brachytherapy $\mathrm{CB}=$ ciliary body; Malign = malignant; $\mathrm{NA}=$ nonavailable; $\mathrm{NOS}=$ not otherwise specified; $\mathrm{OD}=$ right eye; OS = left eye; $\mathrm{SCM}=$ spindle cell melanoma.

TABLE 4. Risk of Second Primary Neoplasms by Site, According to Time Since the Diagnosis of Uveal Melanoma

\begin{tabular}{|c|c|c|c|c|c|c|c|c|c|c|}
\hline \multirow[b]{3}{*}{ Cancer Site } & \multicolumn{10}{|c|}{ Time Since Diagnosis } \\
\hline & \multicolumn{2}{|r|}{ 2-11 Months } & \multicolumn{2}{|r|}{ 1-4 Years } & \multicolumn{2}{|r|}{ 5-9 Years } & \multicolumn{2}{|r|}{$10-19$ Years } & \multicolumn{2}{|r|}{$\geq 20$ Years } \\
\hline & O & O/E (95\% Cl) & 0 & O/E (95\% Cl) & 0 & O/E (95\% Cl) & 0 & O/E $(95 \% \mathrm{Cl})$ & 0 & O/E (95\% Cl) \\
\hline All sites & & $1.29(0.97-1.68)$ & 203 & $1.23^{a}(1.07-1.42)$ & 151 & $1.06(0.90-1.25)$ & 175 & $1.08(0.93-1.26)$ & 58 & $0.88(0.67-1.14)$ \\
\hline $\begin{array}{l}\text { All sites except } \\
\text { UM }\end{array}$ & & $1.24(0.93-1.63)$ & 200 & $1.22^{a}(1.05-1.40)$ & 147 & $1.04(0.87-1.22)$ & 174 & $1.08(0.93-1.25)$ & 55 & $0.84(0.63-1.09)$ \\
\hline Salivary gland & & $($ Expected $=0.10)$ & & $10.16^{a}(2.77-26.00)$ & 1 & $2.94(0.07-16.39)$ & & $($ Expected $=0.40)$ & 1 & $5.66(0.14-31.54)$ \\
\hline Soft tissue & & $($ Expected $=0.19)$ & 3 & $4.07(0.84-11.90)$ & 1 & $1.58(0.04-8.83)$ & 3 & $4.07(0.84-11.88)$ & 1 & $2.93(0.07-16.32)$ \\
\hline Skin melanoma & & $4.39^{a}(1.61-9.56)$ & 25 & $4.59^{a}(2.97-6.77)$ & 10 & $2.10^{a}(1.01-3.86)$ & 14 & $2.52^{a}(1.38-4.23)$ & 3 & $1.12(0.23-3.27)$ \\
\hline Kidney & & $1.06(0.03-5.90)$ & 11 & $2.95^{a}(1.47-5.27)$ & 6 & $1.85(0.68-4.02)$ & & $2.44^{a}(1.12-4.63)$ & 1 & $0.62(0.02-3.43)$ \\
\hline $\begin{array}{l}\text { Eye/orbit } \\
\text { melanoma }\end{array}$ & & $32.56^{a}(3.94-117.61)$ & & $12.88^{a}(2.66-37.63)$ & & $21.12^{a}(5.76-54.09)$ & & $4.91(0.12-27.35)$ & & $36.72^{a}(7.57-107.32)$ \\
\hline $\begin{array}{r}\mathrm{Cl}=\text { confiden } \\
\text { Only sites witr } \\
\text { When observe } \\
\text { above analyses. } \\
{ }^{a} 95 \% \mathrm{Cl} \text { not ir }\end{array}$ & a sig & $\begin{array}{l}\text { interval; } \mathrm{O}=\text { observed } \\
\text { gnificantly higher stand } \\
\text { /alues were equal to } 0, \mathrm{e} \\
\text { dding the null value }(=1\end{array}$ & $\begin{array}{l}\text { numb } \\
\text { dardiz } \\
\text { expec }\end{array}$ & $\begin{array}{l}\text { ber of cases; O/E ra } \\
\text { ed incidence rates } f \\
\text { ted values were rep } \\
P<.05 \text {. }\end{array}$ & for pa & $\begin{array}{l}\text { observed over expe } \\
\text { tients with uveal me } \\
\text { No correction for m }\end{array}$ & $\begin{array}{l}\text { ected } \\
\text { elanor } \\
\text { nultipl }\end{array}$ & $\begin{array}{l}\text { number of cases; } \\
\text { ma are presented. } \\
\text { le comparisons was }\end{array}$ & $\begin{array}{l}\text { UM } \\
\text { sper }\end{array}$ & $\begin{array}{l}=\text { uveal melanoma. } \\
\text { formed for any of the }\end{array}$ \\
\hline
\end{tabular}

of follow-up and later among 10- to 19-year survivors. Skin melanoma risk was increased throughout, and was still significant in 19-year survivors.

Considering the relevance of age and sex for cancer in general, we additionally evaluated SIRs stratified by these parameters. Our results revealed that subjects aged 30-59 years at the time of diagnosis of uveal melanoma presented the higher risk of developing SPN (for all sites, SIR = 1.18; 95\% CI: 1.04-1.34). Interestingly, those diagnosed before that age did not present a significantly increased risk compared to the reference population (Table 5). Regarding sex, compared to the female reference population, women with a previous uveal melanoma presented a $17 \%$ significantly increased risk of all sites SPN (95\% CI: 1.04-1.32, Table 6). There was a trend towards an increased risk for men $(7 \%)$, but it was not significant. For both sexes, the described significantly higher risk of skin melanoma and kidney tumors was still observed.

- TREATMENT FOR THE FIRST MALIGNANCY: Thirty-nine percent of the patients $(\mathrm{n}=1538)$ received radiation therapy, including a small group who underwent both 
TABLE 5. Risk of Second Primary Neoplasms in Uveal Melanoma Patients, by Age Category at Diagnosis

\begin{tabular}{|c|c|c|c|c|c|c|c|c|c|c|c|c|}
\hline \multirow[b]{3}{*}{ Second cancer site } & \multicolumn{12}{|c|}{ Age at Diagnosis of Uveal Melanoma } \\
\hline & \multicolumn{4}{|c|}{$<30$ Years } & \multicolumn{4}{|c|}{$30-59$ Years } & \multicolumn{4}{|c|}{$\geq 60$ Years } \\
\hline & 0 & O/E & $95 \% \mathrm{Cl}$ & EAR & 0 & $\mathrm{O} / \mathrm{E}$ & $95 \% \mathrm{Cl}$ & EAR & 0 & O/E & $95 \% \mathrm{Cl}$ & EAR \\
\hline All sites & 5 & 1.16 & $0.36-2.72$ & 3.22 & 254 & $1.18^{a}$ & $1.04-1.34$ & 19.80 & 382 & 1.07 & $0.97-1.18$ & 16.04 \\
\hline All sites excluding second UM & 5 & 1.17 & $0.38-2.72$ & 3.27 & 246 & $1.15^{a}$ & $1.01-1.30$ & 15.98 & 377 & 1.06 & $0.95-1.17$ & 13.16 \\
\hline Salivary gland & 0 & 0.00 & $0.00-241.97$ & -0.07 & 2 & 3.81 & $0.46-13.76$ & 0.74 & 4 & $4.60^{a}$ & $1.25-11.79$ & 2.02 \\
\hline Soft tissue including Heart & 0 & 0.00 & $0.00-86.95$ & -0.19 & 2 & 1.93 & $0.23-6.96$ & 0.48 & 6 & $3.86^{a}$ & $1.42-8.39$ & 2.86 \\
\hline Skin melanoma & 1 & 2.40 & $0.06-13.37$ & 2.66 & 27 & $2.95^{a}$ & $1.95-4.29$ & 8.94 & 30 & $2.93^{a}$ & $1.97-4.18$ & 12.72 \\
\hline Kidney & 0 & 0.00 & $0.00-31.14$ & -0.54 & 14 & $2.43^{a}$ & $1.33-4.09$ & 4.13 & 14 & $1.90^{a}$ & $1.04-3.19$ & 4.27 \\
\hline Eye and Orbit-Melanoma & 0 & 0.00 & $0.00-404.39$ & -0.04 & 8 & $24.05^{a}$ & $10.38-47.39$ & 3.84 & 5 & $11.70^{a}$ & $3.80-27.30$ & 2.95 \\
\hline Miscellaneous $^{b}$ & 0 & 0.00 & $0.00-82.47$ & -0.20 & 4 & 1.02 & $0.28-2.61$ & 0.04 & 16 & 1.57 & $0.90-2.54$ & 3.72 \\
\hline $\begin{array}{l}\mathrm{Cl}=\text { confidence interval; EAR } \\
\text { UM = uveal melanoma. } \\
\text { Only sites with significantly h } \\
\text { When observed values were } \\
\text { above analyses. } \\
{ }^{a} 95 \% \text { confidence interval not } \\
{ }^{b} \text { Miscellaneous represents all } \\
\text { and codes available at http://se }\end{array}$ & ghe & $\begin{array}{l}\text { kcess a } \\
\text { stand } \\
\text { to } 0, \mathrm{e} \\
\text { uding t } \\
\text { and his } \\
\text { ancer.g }\end{array}$ & $\begin{array}{l}\text { bsolute risk; O } \\
\text { ardized inciden } \\
\text { xpected values } \\
\text { he null value (= } \\
\text { stologic combin } \\
\text { yov/siterecode }\end{array}$ & $\begin{array}{l}=\text { observ } \\
\text { ce rates } \\
\text { were rep } \\
\text { 1), so } P \\
\text { lations nc } \\
\text { b/icdo3 }\end{array}$ & $\begin{array}{l}\text { fed nu } \\
\text { for pa } \\
\text { ported } \\
<.05 \\
\text { ot cov } \\
\text { who2 }\end{array}$ & $\begin{array}{l}\text { mber of c } \\
\text { tients wit } \\
\text { No corre } \\
\text { aned in the } \\
008 /) \text {. }\end{array}$ & $\begin{array}{l}\text { ases; O/E ratio } \\
\text { ction for multip } \\
\text { remaining gro }\end{array}$ & $\begin{array}{l}\text { ma are } \\
\text { le comp }\end{array}$ & $\begin{array}{l}\text { oreser } \\
\text { arison }\end{array}$ & $\begin{array}{l}\text { ted. } \\
\text { s was per }\end{array}$ & formed for an & of the \\
\hline
\end{tabular}

TABLE 6. Risk of Second Primary Neoplasms in Uveal Melanoma Patients, by Sex

\begin{tabular}{|c|c|c|c|c|c|c|c|c|}
\hline \multirow[b]{3}{*}{ Second cancer site } & \multicolumn{8}{|c|}{ Sex } \\
\hline & \multicolumn{4}{|c|}{ Male } & \multicolumn{4}{|c|}{ Female } \\
\hline & 0 & O/E & $95 \% \mathrm{Cl}$ & EAR & 0 & O/E & $95 \% \mathrm{Cl}$ & EAR \\
\hline All sites & 375 & 1.07 & $0.97-1.19$ & 13.08 & 266 & $1.17^{a}$ & $1.04-1.32$ & 21.84 \\
\hline All sites excluding second UM & 366 & 1.05 & $0.94-1.16$ & 8.78 & 262 & $1.16^{a}$ & $1.02-1.31$ & 19.82 \\
\hline Salivary gland & 3 & 3.17 & $0.65-9.26$ & 1.05 & 3 & $6.49^{a}$ & $1.34-18.97$ & 1.40 \\
\hline Soft tissue including heart & 5 & 3.07 & $1.00-7.16$ & 1.72 & 3 & 2.98 & $0.61-8.70$ & 1.10 \\
\hline Skin melanoma & 27 & $2.00^{a}$ & $1.32-2.91$ & 6.89 & 31 & $4.90^{a}$ & $3.33-6.96$ & 13.65 \\
\hline Kidney & 17 & $1.87^{a}$ & $1.09-3.00$ & 4.04 & 11 & $2.64^{a}$ & $1.32-4.72$ & 3.78 \\
\hline Eye and orbit melanoma & 9 & $19.83^{a}$ & $9.11-37.83$ & 4.36 & 4 & $12.60^{a}$ & $3.43-32.25$ & 2.04 \\
\hline Miscellaneous $^{b}$ & 7 & 0.91 & $0.37-1.88$ & -0.35 & 13 & $2.00^{a}$ & $1.07-3.42$ & 3.60 \\
\hline $\begin{array}{l}\mathrm{Cl}=\text { confidence interval; EAR } \\
\text { UM = uveal melanoma. } \\
\text { Only sites with significantly hi } \\
\text { When observed values were e } \\
\text { above analyses. } \\
{ }^{a} 95 \% \mathrm{Cl} \text { not including the nul } \\
{ }^{b} \mathrm{Miscellaneous} \mathrm{represents} \mathrm{all} \\
\text { and codes available at http://se }\end{array}$ & $\begin{array}{l}\text { stanc } \\
\text { to } 0, \epsilon \\
\text { ae }(= \\
\text { and hi }\end{array}$ & $\begin{array}{l}\text { lute risk; } \\
\text { zed incide } \\
\text { cted value } \\
P<.05 \text {. } \\
\text { gic comb } \\
\text { siterecod }\end{array}$ & $\begin{array}{l}\text { observed num } \\
\text { rates for pat } \\
\text { ere reported. }\end{array}$ & $\begin{array}{l}\text { f cases; } \\
\text { with uve } \\
\text { orrection }\end{array}$ & $\begin{array}{l}\text { elanon } \\
\text { nultiple }\end{array}$ & $\begin{array}{l}\text { re present } \\
\text { nparisons }\end{array}$ & performed fc & $\begin{array}{l}\text { of the } \\
\text { all data }\end{array}$ \\
\hline
\end{tabular}

radiotherapy and surgery $(n=209,5 \%)$. Surgery was the only treatment performed in 2127 (54\%) of the patients, and 266 (7\%) of them were not submitted to any treatment (Table 1). Among those who received radiotherapy, most patients were treated with brachytherapy $(\mathrm{n}=885,22 \%)$, followed by beam irradiation $(\mathrm{n}=549,14 \%)$, combination of both $(\mathrm{n}=37,0.9 \%)$, and others not specified $(\mathrm{n}=67,2 \%)$.
In addition to performing SIR calculations for the global population described above, we assessed SPN risk after uveal melanoma according to whether subjects received radiotherapy or not (Table 7). Patients who underwent radiation treatment showed a $17 \%$ statistically significant increased risk of second tumors involving any site compared to the reference population. This risk was $9 \%$ 
TABLE 7. Second Primary Cancer Risk by Site for Uveal Melanoma Patients, According to Previous Radiotherapy Treatment

\begin{tabular}{|c|c|c|c|c|}
\hline \multirow[b]{3}{*}{ Cancer Site } & \multicolumn{4}{|c|}{ Radiation Treatment } \\
\hline & \multicolumn{2}{|r|}{ Yes } & \multicolumn{2}{|r|}{ No } \\
\hline & o & O/E (95\% Cl) & O & O/E $(95 \% \mathrm{Cl})$ \\
\hline All sites & 202 & $1.17^{a}(1.01-1.34)$ & 438 & $1.09(0.99-1.20)$ \\
\hline All sites excluding UM & 193 & $1.12(0.97-1.29)$ & 434 & $1.08(0.98-1.19)$ \\
\hline All solid tumors & 184 & $1.20^{a}(1.03-1.38)$ & 386 & $1.08(0.98-1.20)$ \\
\hline Brain and other nervous system & 3 & $1.62(0.33-4.74)$ & 5 & $1.18(0.38-2.76)$ \\
\hline Oral cavity and pharynx & 3 & $0.75(0.16-2.20)$ & 8 & $0.84(0.36-1.65)$ \\
\hline - Salivary gland & 2 & $4.62(0.56-16.70)$ & 4 & $4.12^{a}(1.12-10.54)$ \\
\hline Digestive system & 32 & $0.97(0.66-1.37)$ & 73 & $0.87(0.68-1.09)$ \\
\hline Liver and biliary tract & 1 & $0.33(0.01-1.86)$ & 8 & $1.24(0.53-2.44)$ \\
\hline Pancreas & 5 & $1.08(0.35-2.53)$ & 8 & $0.73(0.32-1.44)$ \\
\hline Peritoneum, omentum, and mesentery & 2 & $10.35^{a}(1.25-37.39)$ & 0 & $($ Expected $=0.33)$ \\
\hline Endocrine system & 5 & $2.59(0.85-6.05)$ & 6 & $1.79(0.66-3.89)$ \\
\hline Breast & 19 & $0.99(0.60-1.55)$ & 45 & $1.00(0.73-1.34)$ \\
\hline Respiratory system & 23 & $0.83(0.52-1.24)$ & 55 & $0.84(0.63-1.10)$ \\
\hline Soft tissue including heart & 3 & $3.58(0.74-10.45)$ & 5 & $2.80(0.90-6.53)$ \\
\hline All lymphatic and hematopoietic diseases & 12 & $0.82(0.42-1.43)$ & 36 & $1.09(0.76-1.50)$ \\
\hline Skin melanoma & 18 & $2.61^{a}(1.54-4.10)$ & 40 & $3.12^{a}(2.23-4.24)$ \\
\hline Urinary system & 15 & $0.97(0.54-1.60)$ & 51 & $1.48^{a}(1.10-1.95)$ \\
\hline - Kidney & 7 & $1.60(0.64-3.30)$ & 21 & $2.38^{a}(1.47-3.64)$ \\
\hline Female genital system & 12 & $1.61(0.83-2.81)$ & 23 & $1.23(0.78-1.85)$ \\
\hline Male genital system & 40 & $1.18(0.84-1.60)$ & 71 & $0.96(0.75-1.21)$ \\
\hline Eye and orbit melanoma & 9 & $38.35^{a}(17.53-72.79)$ & 4 & $7.58^{a}(2.05-19.24)$ \\
\hline
\end{tabular}

in subjects who did not receive radiation. However, when analyzing SIRs by site, we observed that the significant increase observed in the radiotherapy subgroup was mainly due to an elevated risk of melanomas of both the skin and the eye and tumors of the peritoneum. The increased risk for salivary gland and kidney tumors described above for the entire study population occurred in the group of patients that did not receive radiotherapy. Indeed, when we performed this analysis excluding cases coded as a second uveal melanoma (which, as mentioned, probably represent misclassification of recurrences), the increased risk for all sites in subjects who received radiotherapy compared to the reference population lost its statistical significance $(\mathrm{SIR}=1.12,95 \%$ CI: 0.97-1.29).

To further explore a potential effect of radiation treatment on SPN occurrence, we performed a Cox analysis (Table 8). The univariate model revealed that the hazard of developing a SPN was not significantly higher in patients who had received radiotherapy for uveal melanoma when compared to patients who did not receive this treatment (hazard ratio $[\mathrm{HR}]=1.08,95 \% \mathrm{CI}: 0.90-1.29, P=.39$; Figure). To account for confounding, we also performed a multivariate analysis, which revealed that radiotherapy was not an independent risk factor for SPN, even after adjusting for confounders ( $\mathrm{HR}=1.06,95 \% \mathrm{CI}: 0.88-1.26$, $P=.54$, Table 8$)$. Older age (HR $=1.04,95 \%$ CI: $1.03-$ $1.05, P<.001)$ and male sex $(\mathrm{HR}=0.70,95 \% \mathrm{CI}: 0.59$ $0.82, P<.001$, Table 8 ) were statistically significantly associated with a higher hazard for SPN. Supplemental Figures 1 and 2 present the graphs for these analyses (Supplemental Figures 1 and 2 available at AJO.com). The multivariate analysis was repeated excluding cases coded as a second uveal melanoma and the same results were observed (for radiotherapy, $\mathrm{HR}=1.02,95 \% \mathrm{CI}: 0.85-1.23, P=.80$ ). There was also no association between radiotherapy and late-occurring SPN-when considering only subjects who survived at least 10 years $(\mathrm{n}=1343)$ the HR was 1.00 (95\% CI: 0.70-1.42, $P=.99$ ). Finally, we assessed if the modality of radiotherapy influenced the hazard of developing SPNs. Our multivariate analysis revealed that both beam irradiation $(\mathrm{HR}=0.97,95 \% \mathrm{CI}: 0.75-1.27, \mathrm{P}=.84)$ and brachytherapy $(\mathrm{HR}=1.10,95 \% \mathrm{CI}: 0.88-1.38, P=.40)$ were not significant. When all the previous multivariate models were refit accounting for competing risks, the results were largely unchanged (Supplemental Figure 3 and Supplemental Table, available at AJO.com). 


\begin{tabular}{|c|c|c|c|}
\hline \multicolumn{4}{|c|}{$\begin{array}{l}\text { TABLE 8. Univariate and Multivariate Cox Proportional } \\
\text { Hazard Model Analysis of Second Primary Neoplasms }\end{array}$} \\
\hline \multicolumn{4}{|c|}{ Univariate Analysis } \\
\hline & $\mathrm{HR}$ & $95 \% \mathrm{Cl}$ & $P$ Value \\
\hline Radiotherapy & 1.08 & $0.90-1.29$ & .39 \\
\hline $\operatorname{Sex}^{a}$ & 0.78 & $0.66-0.92$ & $.003^{\star}$ \\
\hline Race group ${ }^{b}$ & 0.98 & $0.44-2.20$ & .97 \\
\hline Age at diagnosis & 1.03 & $1.03-1.04$ & $<.001^{\star}$ \\
\hline \multicolumn{4}{|c|}{ Multivariate Analysis } \\
\hline & $\mathrm{HR}$ & $95 \% \mathrm{Cl}$ & $P$ Value \\
\hline Radiotherapy & 1.06 & $0.88-1.26$ & .54 \\
\hline $\operatorname{Sex}^{a}$ & 0.70 & $0.59-0.82$ & $<.001^{\star}$ \\
\hline Race group ${ }^{b}$ & 0.83 & $0.37-1.86$ & .65 \\
\hline Age at diagnosis & 1.04 & $1.03-1.05$ & $<.001^{\star}$ \\
\hline \multicolumn{4}{|c|}{$\begin{array}{l}\mathrm{Cl}=\text { confidence interval; } \mathrm{HR}=\text { hazard ratio. } \\
\text { Significant } P \text { values }(\alpha<.05) \text { are indicated with an asterisk. } \\
{ }^{a} \text { Reference term for sex }- \text { male. } \\
{ }^{b} \text { Reference term for race group - nonwhite (black/other). }\end{array}$} \\
\hline
\end{tabular}

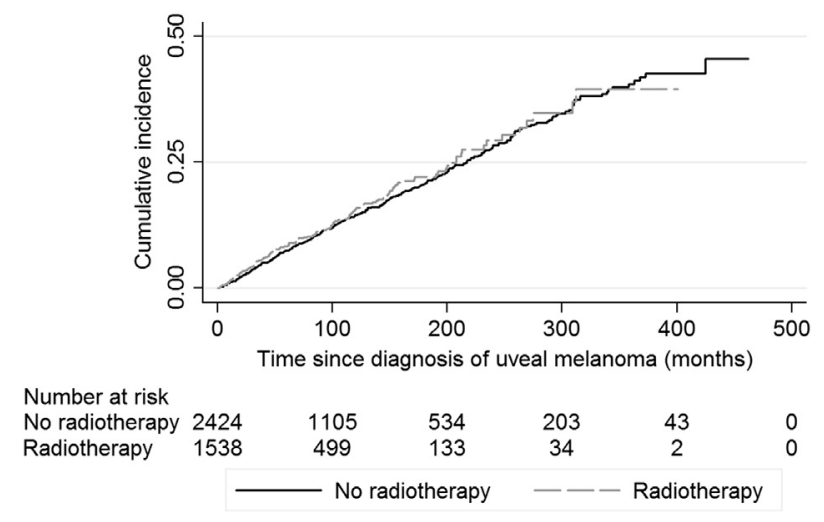

FIGURE. Cumulative risk of developing a second primary neoplasm, according to treatment with radiotherapy.

\section{DISCUSSION}

WE CONDUCTED A POPULATION-BASED STUDY INCLUDING 3976 patients with uveal melanoma and observed that 16\% developed SPNs. This represents an 11\% excess risk compared to the reference SEER population, and was primarily due to patients aged 30-59 years at the time of diagnosis of uveal melanoma. The most common SPN included salivary gland, soft tissue, and kidney tumors, as well as skin melanomas. An increased risk of a second uveal melanoma was also described, but probably represents recurrences. Using multivariate analysis, we verified that radiotherapy was not a significant independent risk factor for SPN.

The observed increased rate of SPN in patients with uveal melanoma is in agreement with other population-based studies performed before radiotherapy became the standard of care. ${ }^{22,24}$ Interestingly, the COMS trial $^{26}$ reported different results, describing that $9.6 \%$ of the patients developed SPNs and that this risk was comparable to the general population. However, this was solely based on a comparison with a historical cohort of American subjects, with a limited age range of 40-59 years. Similarly, Andreoli and associates $^{30}$ recently described absolute numbers of SPN after uveal melanoma, but did not perform any statistical comparisons with their incidence in patients without these tumors. SIR calculations are essential to exclude the role of chance in assessing SPN risk. ${ }^{31}$ Our results are based on these robust statistical comparisons, considering a reference SEER cohort, standardized by age, sex, race, calendar year, and cancer site. We included only subjects with a primary diagnosis of uveal melanoma, thus not considering those who had had other preceding malignancies.

In this study, we also determined that the observed increased risk of SPN in patients with uveal melanoma was primarily due to cancers of the salivary glands, kidney, and soft tissue, as well as skin melanomas. Screening bias may account for the higher observed SIRs of some of these tumors in our cohort, namely those of the salivary glands. This has been described in other malignancies, ${ }^{32}$ as a result of higher detection rates of SPN, or solely their earlier diagnosis, owing to more regular and closer follow-up, mainly in the first years. Indeed, in our study the salivary gland second primaries were mostly diagnosed in the early follow-up, consistent with a screening bias. Furthermore, even though these lesions are located close to the irradiated fields in uveal melanoma and have been linked with radiotherapy, ${ }^{33}$ this was not verified in our analysis, as a higher rate occurred in nonirradiated patients.

Regarding kidney SPN, screening bias has also been postulated. $^{22}$ However, in our series SIR rates were increased not only in the first years, but also with more than 10-year latency. This suggests other underlying mechanisms. The same applies to the observed increased risk of cutaneous melanoma, since it persisted through almost 20 years of follow-up. Moreover, in our cohort all the identified cases of skin melanoma had available histologic confirmation in the SEER database. Considering their distinct pathologic and immunophenotypic features from skin metastasis of uveal melanoma, ${ }^{34,35}$ misclassification is a very unlikely explanation for our findings.

In both cases (kidney SPN and skin melanomas), host susceptibility factors, namely common genetic risk factors, may represent a reasonable explanation. Actually, the recently described tumor predisposition syndrome caused by germline mutations in BAP1 (BRCA1-associated protein) gene confers an increased risk for uveal melanoma, cutaneous melanoma, and renal cell carcinoma, among other malignancies. ${ }^{9-11}$ BAP1 gene is a tumor suppressor gene located in chromosome $3,^{10}$ the most frequently involved in the pathogenesis of uveal melanoma. ${ }^{36}$ This highlights the relevance of considering screening for 
BAP1 somatic mutations in uveal melanoma tumor tissues. Besides the relevance for prognosis, ${ }^{10}$ namely owing to their association with class 2 tumors, ${ }^{15}$ their presence would also raise the possibility of germline mutations, and thus of an increased risk of SPN, which may require further genetic testing and surveillance. Unfortunately, the SEER database does not include any genetic data, namely on gene expression profile, which would be interesting to analyze.

Regarding the observed increased risk of cutaneous melanoma in particular, our findings confirmed those by other groups. ${ }^{22,23,37}$ Despite being rather controversial, the link between skin and uveal melanoma might be their common origin from melanocytes, derived from the neural crest. $^{23}$ However, these 2 tumors show remarkable differences in their metastatic spread and therapeutic response. ${ }^{3,39}$ The same applies to their associated genetic findings: more than $80 \%$ of uveal melanomas carry mutations in GNA11 or GNAQ; in cutaneous melanoma, oncogenic mutations in BRAF and NRAS seem to be primarily involved. ${ }^{15,40,41}$ However, both GNAQ/GNA11 and BRAF/NRAS activate a common pathway, the mitogen-activated protein kinase (MAPK). ${ }^{41}$ Other common mechanisms probably remain to be established.

We also observed in our cohort 13 cases that were coded as a second uveal melanoma, most of them occurring after an initial treatment with radiotherapy. Owing to the nature of our study, we cannot exclude that at least some of these represent late recurrences of the first malignancy. ${ }^{42}$ Additionally, 2 patients were reported to present a melanoma in the fellow eye. One of them was diagnosed 2 months after the primary diagnosis, so most likely represents a bilateral melanoma, ${ }^{43}$ but we cannot exclude the possibility of metastatic disease to the fellow eye. ${ }^{44}$ Considering this, we thought that these cases coded as second independent uveal melanoma could lead to overestimation in our multiple primaries calculations. However, our analysis excluding them confirmed that patients with uveal melanoma presented a significant 9\% excess risk for SPN compared to the SEER reference population.

We additionally evaluated SIR rates stratified by sex and age category. Accordingly to what has been previously described for all SPN combined, ${ }^{45}$ women presented a significantly higher risk of all sites' second malignancies compared to the reference population. Interestingly, for kidney tumors and skin melanoma both sexes presented a significantly excess risk. We did not find any increased risk in our cohort of the typical constellation of hormonedependent tumors (such as breast, uterine corpus, ovary, and prostate). Regarding age at diagnosis, in agreement with the remaining literature for most cancer sites, ${ }^{45}$ we found that the greatest burden of SPN was experienced by patients initially diagnosed with uveal melanoma between 30 and 59 years old. Subjects with an earlier diagnosis $(<30$ years) did not present an increased risk of developing SPN compared to the reference population. Additionally,
SPNs were not diagnosed earlier in our study population than what has been described for these malignancies in general. ${ }^{46}$ This suggests that, despite being a reasonable and important explanation, tumor predisposition syndromes probably occur infrequently. ${ }^{12}$ One also needs to consider that lifestyle and environmental exposures also play a role in SPN risk and these tend to accumulate with life. ${ }^{4}$

Finally, we also focused on the potential influence of the treatment modalities for uveal melanoma on the risk of SPN. In our cohort, SIR calculations according to previous radiotherapy revealed that, after excluding cases coded as a second UM, eyes that had received this treatment did not present a significantly increased all-sites risk as compared to the reference population. Indeed, our multivariate analysis confirmed that radiotherapy was not a significant predictor of SPN occurrence after uveal melanoma, even after adjusting for age, sex, and race. The selection of predictors for this model was based on known clinical risk factors. Tumor stage and size have been considered as potential confounders ${ }^{47}$ but were not included, since we lacked these data for most patients. The same applies to the histologic subtype, as most tumors were classified as "melanoma not otherwise specified." Our observation that radiotherapy is not significantly linked with a higher risk for SPN can be due to a more localized effect of this treatment for ocular melanomas, considering the low volumes irradiated and the modalities applied, ${ }^{42}$ namely their low side-scatter effect. Indeed, the majority of the patients received brachytherapy that is highly localized. ${ }^{48}$ The second most common modality was beam irradiation, which most likely represents proton beam irradiation for most tumors. Owing to the Bragg peak effect, proton beam irradiation-charged particles also have minimal scatter and deliver the maximum dose at the end of their range, ${ }^{49}$ resulting in no radiation exposure to tissues outside the orbit. Indeed, none of the treatment modalities proved to be associated with an increased risk of SPNs. Interestingly, the COMS group $^{26}$ also found no differences in the incidence of SPN in patients who received radiotherapy compared to those who did not receive this treatment. One could argue that the effects of radiation treatment in SPN incidence may take up to 10 years to be evident. ${ }^{13}$ Therefore, we also performed a multivariate analysis only including subjects who survived at least 1 decade and confirmed that radiotherapy was also not significantly associated with a higher risk of SPN in these patients.

The main limitations of our study are its retrospective nature and the inherent limitations of public available databases, such as underreporting (eg, surgical treatment type specification is not available) and the lack of some variables (eg, radiotherapy dosage). Unreliable data on tumor size also precluded including this important parameter in our analyses (sizes not compatible with the eyeball dimensions were described for a significant number of tumors). Additionally, limitations of coding made it difficult to interpret some findings-for example, the observed 
increased risk of soft tissue tumors. The designation "soft tissues" includes a large variety of tumor types that may occur in multiple body sites and are not specified. Additionally, melanoma metastases can histologically mimic some soft tissue tumors, which can lead to misdiagnosis. Moreover, the SEER database does not collect data on genetic and lifestyle risk factors (eg, smoking) that are currently known to influence the risk of $\mathrm{SPN}^{4}$ and would be interesting to analyze. In assessing the incidence of multiple types of SPN, multiple tests were completed that could have led to false-positive associations. Therefore, these results should be interpreted cautiously. Additionally, one must consider that subjects may change their residence during follow-up and subsequent cancers were not recorded in the SEER database for patients who migrate from their original SEER geographic area, regardless of whether they move into another SEER area. This might lead to an underestimation of our results on the incidence of SPN. ${ }^{45}$ Despite these limitations, the SEER database has been well validated ${ }^{3,50}$ and provides a large population for analysis, minimizing the selection bias inherent to single-hospital or clinical series. Uveal melanoma is the most common intraocular malignant tumor, but is relatively rare in the wider context of cancer, so the large size of the SEER database, as well as its long available follow-up, allowed for adequately powered analyses.

In conclusion, this population-based study showed that patients with uveal melanoma demonstrated an $11 \%$ excess risk of developing SPNs compared to the reference population. The most common and biologically meaningful were skin melanomas and kidney tumors, but an increased risk for salivary gland and soft tissue malignancies was also identified. Radiation therapy did not seem to significantly influence the occurrence of SPNs, which can be due to the radiation modalities applied for uveal melanoma and the small volumes irradiated. Host susceptibility, namely genetic risk factors, and lifestyle profiles may be potential explanations for the observed increased risks. With ongoing research to improve the survival of patients with uveal melanoma, assessing and understanding the risk of SPNs will become increasingly important in the followup of these patients.

FUNDING/SUPPORT: INÊS LAÍNS WAS FINANCIALLY SUPPORTED BY THE HARVARD MEDICAL SCHOOL (HMS) PORTUGAL PROgram and the Portuguese Foundation for Science and Technology (FCT) (HMSP-ICJ/0006/2013). This project was initially developed in the context of the Clinical Scholars' Research Training Program (CSRT) of the HMS Portugal Program. Financial disclosures: Brian Healy currently has grants from Merck Serono, Novartis, and Genzyme. Ivana K. Kim received personal fees from Alcon, Inc; Allergan, Inc; Biophytis SA; Castle Biosciences, Inc; and Iconic Therapeutics, Inc. She also received research support from Genentech, Inc. Joan W. Miller received personal fees from Alcon Research Council; Amgen, Inc; Biogen Idec, Inc; Imagen Biotech, Inc; ISIS Pharmaceuticals, Inc; KalVista Pharmaceuticals, Ltd; Maculogix, Inc; and Regeneron Pharmaceuticals, Inc. She also received nonfinancial support from Genentech, Inc; Maculogix, Inc; and ONL Therapeutics, LLC. Currently she has a grant from Lowy Medical Research Institute, Ltd. The following authors have no financial disclosures: Inês Laíns, Carla Bartosch, Vera Mondim, Ivana Kim, and Deeba Husain. All authors attest that they meet the current ICMJE criteria for authorship.

The authors would like to thank the SEER*Stat technical support.

\section{REFERENCES}

1. Kroll S, Char DH, Quivey J, Castro J. A comparison of causespecific melanoma mortality and all-cause mortality in survival analyses after radiation treatment for uveal melanoma. Ophthalmology 1998;105(11):2035-2045.

2. Kujala E, Mäkitie T, Kivelä T. Very long-term prognosis of patients with malignant uveal melanoma. Invest Ophthalmol Vis Sci 2003;44(11):4651-4659.

3. Singh AD, Turell ME, Topham AK. Uveal melanoma: trends in incidence, treatment, and survival. Ophthalmology 2011; 118(9):1881-1885.

4. Morton LM, Onel K, Curtis RE, Hungate EA, Armstrong GT. The rising incidence of second cancers: patterns of occurrence and identification of risk factors for children and adults. Am Soc Clin Oncol Educ Book 2014;34:e57-67.

5. Morton LM, Swerdlow AJ, Schaapveld M, et al. Current knowledge and future research directions in treatment-related second primary malignancies. Eur J Cancer Suppl 2014;12(1):5-17.

6. Vineis P, Alavanja M, Buffler P, et al. Tobacco and cancer: recent epidemiological evidence. J Natl Cancer Inst 2004; 96(2):99-106.

7. Boffetta P, Hashibe M, La Vecchia C, Zatonski W, Rehm J. The burden of cancer attributable to alcohol drinking. Int J Cancer 2006;119(4):884-887.
8. Oeffinger KC, Baxi SS, Novetsky Friedman D, Moskowitz CS. Solid tumor second primary neoplasms: who is at risk, what can we do? Semin Oncol 2013;40(6):676-689.

9. Njauw C-NJ, Kim I, Piris A, et al. Germline BAP1 inactivation is preferentially associated with metastatic ocular melanoma and cutaneous-ocular melanoma families. PLoS One 2012; 7(4):e35295.

10. Battaglia A. The importance of multidisciplinary approach in early detection of BAP1 tumor predisposition syndrome: clinical management and risk assessment. Clin Med Insights Oncol 2014;8:37-47.

11. Cheung M, Talarchek J, Schindeler K, et al. Further evidence for germline BAP1 mutations predisposing to melanoma and malignant mesothelioma. Cancer Genet 2013;206(5): 206-210.

12. Gupta MP, Lane AM, DeAngelis MM, et al. Clinical characteristics of uveal melanoma in patients with germline BAP1 mutations. JAMA Ophthalmol 2015;133(8):881-887.

13. Chaturvedi AK, Engels EA, Gilbert ES, et al. Second cancers among 104,760 survivors of cervical cancer: evaluation of long-term risk. J Natl Cancer Inst 2007;99(21):1634-1643.

14. Grantzau T, Overgaard J. Risk of second non-breast cancer after radiotherapy for breast cancer: a systematic review and meta-analysis of 762,468 patients. Radiother Oncol 2015; 114(1):56-65. 
15. Jovanovic P, Mihajlovic M, Djordjevic-Jocic J, Vlajkovic S, Cekic S, Stefanovic V. Ocular melanoma: an overview of the current status. Int J Clin Exp Pathol 2013;6(7):1230-1244.

16. The American Brachytherapy Society consensus guidelines for plaque brachytherapy of uveal melanoma and retinoblastoma. Brachytherapy 2014;13(1):1-14.

17. Kumar S, Shah JP, Bryant CS, et al. Second neoplasms in survivors of endometrial cancer: impact of radiation therapy. Gynecol Oncol 2009;113(2):233-239.

18. Hei TK, Zhou H, Chai Y, Ponnaiya B, Ivanov VN. Radiation induced non-targeted response: mechanism and potential clinical implications. Curr Mol Pharmacol 2011;4(2):96-105.

19. Lorimore SA, Coates PJ, Wright EG. Radiation-induced genomic instability and bystander effects: inter-related nontargeted effects of exposure to ionizing radiation. Oncogene 2003;22(45):7058-7069.

20. Tomita M, Maeda M. Mechanisms and biological importance of photon-induced bystander responses: do they have an impact on low-dose radiation responses. J Radiat Res 2015; 56(2):205-219.

21. Morgan WF, Sowa MB. Non-targeted effects induced by ionizing radiation: mechanisms and potential impact on radiation induced health effects. Cancer Lett 2015;356(1):17-21.

22. Scélo G, Boffetta P, Autier P, et al. Associations between ocular melanoma and other primary cancers: an international population-based study. Int J Cancer 2007;120(1):152-159.

23. Shors AR, Iwamoto S, Doody DR, Weiss NS. Relationship of uveal and cutaneous malignant melanoma in persons with multiple primary tumors. Int J Cancer 2002;102(3):266-268.

24. Bergman L, Nilsson B, Ragnarsson-Olding B, Seregard S. Uveal melanoma: a study on incidence of additional cancers in the Swedish population. Invest Ophthalmol Vis Sci 2006; $47(1): 72-77$.

25. Swerdlow AJ, Storm HH, Sasieni PD. Risks of second primary malignancy in patients with cutaneous and ocular melanoma in Denmark, 1943-1989. Int J Cancer 1995;61(6):773-779.

26. Diener-West M, Reynolds SM, Agugliaro DJ, et al. Second primary cancers after enrollment in the COMS trials for treatment of choroidal melanoma: COMS Report No. 25. Arch Ophthalmol 2005;123(5):601-604.

27. Surveillance, Epidemiology, and End Results (SEER) Program Research Data (1973-2011), National Cancer Institute, DCCPS, Surveillance Research Program, Surveillance Systems Branch, Released April 2014. Available at www.seer. cancer.gov. Based on the November 2013 Submission.

28. Hayat MJ, Howlader N, Reichman ME, Edwards BK. Cancer statistics, trends, and multiple primary cancer analyses from the Surveillance, Epidemiology, and End Results (SEER) Program. Oncologist 2007;12(1):20-37.

29. Surveillance, Epidemiology and ERD (SEER). US Population Data - 1969-2013. Available at http://seer.cancer.gov/popdata/. Accessed November 5, 2015.

30. Andreoli MT, Mieler WF, Leiderman YI. Epidemiological trends in uveal melanoma. Br J Ophthalmol 2015;99(11): 1550-1553.

31. Soerjomataram I, Willem Coebergh J. Epidemiology of multiple primary cancers. In: Verma M, ed. Cancer Epidemiology - Volume I, Host Susceptibility Factors. Bethesda, Maryland: Humana Press; 2009:85-106.
32. Van Hemelrijck M, Drevin L, Holmberg L, Garmo H, Adolfsson J, Stattin P. Primary cancers before and after prostate cancer diagnosis. Cancer 2012;118(24):6207-6216.

33. Sun EC, Curtis R, Melbye M, Goedert JJ. Salivary gland cancer in the United States. Cancer Epidemiol Biomarkers Prev 1999;8(12):1095-1100.

34. Guerriere-Kovach PM, Hunt EL, Patterson JW, Glembocki DJ, English JC, Wick MR. Primary melanoma of the skin and cutaneous melanomatous metastases: comparative histologic features and immunophenotypes. Am J Clin Pathol 2004;122(1):70-77.

35. Iwamoto S, Burrows RC, Kalina RE, et al. Immunophenotypic differences between uveal and cutaneous melanomas. Arch Ophthalmol 2002;120(4):466-470.

36. Nagarkatti-Gude N, Wang Y, Ali MJ, Honavar SG, Jager MJ, Chan C-C. Genetics of primary intraocular tumors. Ocul Immunol Inflamm 2012;20(4):244-254.

37. van Hees CL, Jager MJ, Bleeker JC, Kemme H, Bergman W. Occurrence of cutaneous and uveal melanoma in patients with uveal melanoma and their first degree relatives. Melanoma Res 1998;8(2):175-180.

38. Singh P, Singh A. Choroidal melanoma. Oman J Ophthalmol 2012;5(1):3-9.

39. van den Bosch T, Kilic E, Paridaens D, de Klein A. Genetics of uveal melanoma and cutaneous melanoma: two of a kind? Dermatol Res Pract 2010;2010:360136.

40. Schoenfield L. Uveal melanoma: a pathologist's perspective and review of translational developments. Adv Anat Pathol 2014;21(2):138-143.

41. Patel SP. Latest developments in the biology and management of uveal melanoma. Curr Oncol Rep 2013;15(6): 509-516.

42. Finger PT. Radiation therapy for choroidal melanoma. Surv Ophthalmol 1997;42(3):215-232.

43. Hadden PW, Damato BE, McKay IC. Bilateral uveal melanoma: a series of four cases. Eye (Lond) 2003;17(5):613-616.

44. Bourla DH, Young TA. Treatment considerations for primary uveal melanoma with choroidal metastasis to the fellow eye. Semin Ophthalmol 2007;22(3):175-177.

45. Fraumeni JF, Curtis RE, Edwards BK, Tucker MA. Chapter 1, Introduction. In: Curtis RE, Freedman DM, Ron E, et al., eds. New Malignancies Among Cancer Survivors: SEER Cancer Registries, 1973-2000. No. 05-530. Bethesda, Maryland: National Cancer Institute, NIH Publ.; 2006:1-15.

46. Surveillance, Epidemiology and ERD (SEER). SEER Cancer Statistics Review 1975-2012. Available at http://seer. cancer.gov/csr/1975_2012/results_merged/topic_med_age.pdf. Accessed November 5, 2015.

47. Moon K, Stukenborg GJ, Keim J, Theodorescu D. Cancer incidence after localized therapy for prostate cancer. Cancer 2006;107(5):991-998.

48. Stannard C, Sauerwein W, Maree G, Lecuona K. Radiotherapy for ocular tumours. Eye 2012;27(2):119-127.

49. Gragoudas ES, Marie Lane A. Uveal melanoma: proton beam irradiation. Ophthalmol Clin North Am 2005;18(1):111-118. ix.

50. Inskip PD. Multiple primary tumors involving cancer of the brain and central nervous system as the first or subsequent cancer. Cancer 2003;98(3):562-570. 